\title{
Modelo Matemático Aplicado para Imunologia de HIV $^{1}$
}

G.I. POLI ${ }^{2}$ H.M. YANG ${ }^{3}$, Departamento de Matemática Aplicada, Instituto de Matemática, Estatística e Computação Científica, UNICAMP e Lab-Epifisma, Cx.P. 6065, 13081-970 Campinas, SP, Brasil.

Resumo. Neste trabalho desenvolvemos e analisamos um modelo simples que descreve a interação do vírus HIV com o sistema imunológico (SI). O modelo considera apenas a ação de anticorpos para neutralizar vírus HIV circulante no sangue. Obtendo-se o valor de bifurcação, discute-se a possibilidade de SI eliminar HIV circulante.

\section{Introdução}

O estudo e a compreensão do sistema imunológico foram impulsionados extraordinariamente durante as duas últimas décadas como parte essencial do esforço de pesquisa sobre AIDS. O conhecimento resultante, todavia, deverá ter um impacto muito mais abrangente, em particular, e de maneira especial, no aperfeiçoamento das estratégias de tratamento e prevenção de doenças infecciosas estudadas pela epidemiologia. Os modelos matemáticos têm sido usados com bastante sucesso para descrever a transmissão de micro ou macro-parasitas [1] [2] [3] [4]. A utilização de modelos matemáticos e estatísticos em fenômenos bio-médicos tem como finalidade principal ajudar na compreensão dos mecanismos de propagação [7] [6]. Neste sentido, é preciso analisar o sistema de equações resultante da modelagem matemática tanto estático (estabilidade dos pontos de equilíbrio) como dinamicamente (trajetórias do sistema). Uma vez tendo demonstrado que o modelo proposto descreve com certo realismo o fenômeno biológico, o mesmo pode vir a nortear pesquisas posteriores.

A síndrome de imunodeficiência adquirida (AIDS) é uma das doenças infecciosas que mais mata no mundo, em especial adultos jovens. Segundo dados da Organização Mundial de Saúde (OMS), 40 milhões de pessoas são infectadas. A AIDS não tem cura e já matou cerca de 20 milhões de pessoas desde o início da epidemia. Em geral a incidência de AIDS é mais intensa em regiões onde a infra-estrutura para prevenção e tratamento é muito limitada. A doença é causada pelo vírus HIV (vírus da imunodeficiência humana), que ataca as células do sistema imunológico, impedindo uma resposta adequada a um ataque externo como, por exemplo, bactérias e

\footnotetext{
${ }^{1}$ Apoio financeiro Fapesp (Projeto temático) e CNPq (Edital BIC e Edital Universal)

${ }^{2}$ Bolsista BIC (poli@ime.unicamp.br)

${ }^{3}$ Bolsista CNPq (hyunyang@ime.unicamp.br)
} 
outros vírus. Com a progressão da doença, o organismo torna-se mais suscetível às doenças oportunistas, fazendo com que o indivíduo morra devido a essas infecções secundárias. O HIV sofreu algumas modificações genéticas, desde que passou do macaco para o homem, formando diferentes subtipos de vírus. O HIV1 é o causador da epidemia mundial de AIDS e pode ser dividido em três grupos: $\mathrm{M}$, O e N. O grupo M é o mais abundante no mundo e evoluiu geneticamente para formar subtipos que vão de A a J. No Brasil, encontramos o subtipo B como predominante (80\% das infecções), seguidos dos subtipos F e C (com maior prevalência na região Sul do Brasil). O HIV2 ocorre na África Subsaariana, região onde a doença evolui mais rapidamente.

O sistema imunológico é dividido em dois sistemas principais: o primeiro é o sistema imunológico inato - inato no sentido de que o corpo nasce com a habilidade de reconhecer certos micróbios imediatamente e destrui-los -, e o segundo é o sistema imunológico adaptativo, no qual os anticorpos ocupam um papel fundamental. O sistema imunológico inato pode destruir muitos patógenos no primeiro encontro, porém a imunidade inata não pode proteger contra todas as infecções. Aqueles micróbios que se desenvolvem muito rapidamente não podem ser combatidos unicamente pelas defesas inatas do organismo, as quais, comparativamente, desenvolvem-se lentamente. O sistema imunológico adaptativo habilita o organismo a reconhecer e a responder contra qualquer tipo de micróbio, mesmo que nunca tenha tido contato prévio. Este sistema compreende dois tipos de respostas: uma, dita imunidade humoral e a outra, imunidade celular. A primeira ocorre através da ação de anticorpos, os quais são produzidos por linfócitos denominados células B, as quais são formadas na medula óssea (bone marrow) e é particularmente importante no combate a patógenos circulantes na corrente sangüínea. A segunda envolve linfócitos que se originam no timo e por isso são chamados de células T. Estas são divididas em dois tipos, as células T-CD4 e T-CD8, estas últimas sendo chamadas de células $\mathrm{T}$ citotóxicas por destruirem células infectadas. As células T citotóxicas são fundamentais para combater infecções virais.

A infecção com vírus HIV resulta em uma evolução característica no indivíduo. A uma fase inicial de elevada viremia, que compreende os primeiros seis meses após a infecção, segue-se fase assintomática, de baixa virema, que dura alguns anos. Finalmente, com o vírus conseguindo vencer o sistema imunológico, manifesta-se o comprometimento da resposta imunológica. Para estudar a ação de sistema imunológico, desenvolve-se um modelo matemático considerando apenas a ação de anticorpos para neutralizar vírus HIV circulante no sangue. A análise do modelo em equilíbrio estacionário fornece dois pontos, cujas estabilidades são determinadas por um parâmetro de bifurcação. Na secção 2 propomos e estudamos um modelo matemático, com discussão e conclusão dadas na secção 3 .

\section{Modelo Matemático}

Neste trabalho procuramos desenvolver e analisar um modelo simples que descreve a interação do vírus HIV com as células do sistema imunológico. Quando um indivíduo é infectado por vírus HIV em concentração acima da que o sistema inato 
possa controlar, ocorre a disseminação na corrente sangüínea, onde também circulam os linfócitos. Em especial os linfócitos T-CD4 têm na sua superfície receptores para HIV, pelos quais o vírus penetra no interior da célula. Dentro da célula ocorre a multiplicação do vírus, e consequente liberação com morte celular. Mas o sistema imunológico responde a essa infecção, em que células T-CD4 são estimuladas por células apresentadoras de antígenos que, por sua vez, ativam as células B. Essas células plasmas diferenciam-se e se proliferam, produzindo e liberando anticorpos na corrente sangüínea que neutralizam vírus HIV circulantes. O problema da infecção por HIV é justamente o alvo do vírus ser a célula que auxilia na ativação tanto de células B para produzir anticorpos quanto de células T-CD8 responsáveis pela destruição de células infectadas.

O modelo que desenvolvemos estuda apenas a destruição de vírus HIV circulantes na corrente sangüínea pela ação de anticorpos. A produção de anticorpos por parte das células B ativadas é considerada indiretamente, isto é, a produção depende de células T-CD4 que ativam as células B. As células T-CD4 quando infectadas tornamse fábricas de produção de vírus HIV, que são liberados na corrente sangüínea com a morte celular. Não consideramos nenhum tipo de tratamente.

Assim, o modelo considera apenas concentração de vírus HIV na corrente sangüínea, e populações de células T-CD4 não infectadas responsáveis pela ativação na produção de anticorpos por células B, e de células T-CD4 infectadas por vírus HIV. A dinâmica da interação entre células do sistema imunológico com o vírus de HIV considera as seguintes populações:

1. $v(t)$, número de vírus livres no instante de tempo $t$,

2. $y(t)$, número de células suscetíveis ou não infectadas do sistema imunológico no instante de tempo $t$, e

3. $z(t)$, número de células infectadas no instante de tempo $t$.

A interação entre células do sistema imunológico com vírus é descrito pelos seguintes parâmetros do modelo:

1. $b$, taxa de replicação defeituosa do vírus,

2. a, quantidade de vírus liberada pelas células T-CD4 infectadas quando morrem,

3. $u$, taxa de mortalidade natural das células T-CD4 ( $y$ e $z)$,

4. $\gamma$, taxa de eliminação de vírus circulante por anticorpos produzidos pelas células $\mathrm{B}$, ativadas pelas células T-CD4,

5. $u_{1}$, taxa de mortalidade adicional de células T-CD4 infectadas pelo vírus,

6. $\alpha$, taxa de produção das células T-CD4 suscetíveis, e

7. $\beta$, taxa pela qual as células T-CD4 suscetíveis são infectadas, atráves do contato com o vírus. 
O modelo supõe-se que:

(i) O vírus cresce a uma taxa proporcional ao número de novos vírus replicados no interior das células: $a\left(u+u_{1}\right) z$.

(ii) As células não infectadas do sistema imunológico decrescem por dois motivos:

(ii.1) Por morte natural, a uma taxa proporcional ao número de células $y$ : $-u y$.

(ii.2) Pela infeção causada pelo vírus, ou seja, a uma taxa proporcional ao produto de densidades das células $\mathrm{T}$ e dos vírus livres no organismo: $-\beta y v$.

(iii) As células infectadas crescem à mesma taxa com a qual as células não infectadas decrescem: $\beta v y$. Para o encontro entre vírus e células e consequente infecção, utiliza-se a lei da ação das massas.

(iv) As células infectadas decrescem a uma taxa proporcional a $z:\left(u+u_{1}\right) z$.

Assim a dinâmica entre o vírus e o sistema imunológico pode ser representada pelo sistema de equações diferenciais ordinárias

$$
\left\{\begin{array}{l}
\frac{d v}{d t}=a\left(u+u_{1}\right) z-\gamma y v-b v \\
\frac{d y}{d t}=\alpha-u y-\beta v y \\
\frac{d z}{d t}=\beta v y-u z-u_{1} z .
\end{array}\right.
$$

O sistema dinâmico (2.1) apresenta dois pontos de equilíbrio. O primeiro ponto analisado é o ponto trivial $P_{0}$, com as coordenadas dadas pelos valores

$$
\left\{\begin{array}{l}
v=0 \\
y=\frac{\alpha}{u} \\
z=0 .
\end{array}\right.
$$

Esse ponto representa a ausência (sem contato com vírus ou infecção debelada) de vírus, e a população consiste apenas de células T-CD4 suscetíveis.

A estabilidade do equilíbrio trivial $P_{0}$ é determinada pelos autovalores associados à matriz Jacobiana (linearização do sistema dinâmico em torno do valor de equilíbrio) $J$ calculada com as coordenadas dadas em (2.2), dada por

$$
J=\left(\begin{array}{ccc}
-\left(b+\frac{\alpha \gamma}{u}\right) & 0 & a\left(u+u_{1}\right) \\
-\frac{\beta \alpha}{u} & -u & 0 \\
\frac{\beta \alpha}{u} & 0 & -\left(u+u_{1}\right)
\end{array}\right)
$$

Os autovalores são as soluções da equação característica $\operatorname{det}(J-I \lambda)=0$, onde $I$ é a matriz identidade $3 \times 3$.

Calculando o polinômio característico, temos:

$$
(-u-\lambda)\left[\lambda^{2}+\left(b+u+\frac{\alpha \gamma}{u}+u_{1}\right) \lambda+b\left(u+u_{1}\right)-a \alpha \beta\left(1+\frac{u_{1}}{u}\right)+\alpha \gamma\left(1+\frac{u_{1}}{u}\right)\right]=0 .
$$


O valor de um dos autovalores do polinômio é facilmente obtido:

$$
\lambda=-u \text {. }
$$

Em vez de calcular os valores dos outros dois autovalores associados ao polinômio, utiliza-se o critério de Routh-Hurwitz [5] [8]. Segundo o critério de Routh-Hurwitz, para que o polinômio de ordem 2 , por exemplo, $\lambda^{2}+a_{1} \lambda+a_{2}=0$, tenha raízes com parte real negativa, devemos ter: $a_{1}>0$ e $a_{2}>0$. Se todas as raízes reais forem negativas, ou se todas as raízes tiverem parte real negativa, então o ponto de equilíbrio é localmente e assintoticamente estável.

Aplicando ao polinômio entre colchetes de (2.3) o primeiro critério de RouthHurwitz, $a_{1}>0$, tem-se

$$
b+u+\frac{\alpha \gamma}{u}+u_{1}>0
$$

que é sempre satisfeito, pois todas as variáveis do modelo são positivas. Para a segunda condição $a_{2}>0$, tem-se

$$
b\left(u+u_{1}\right)-a \alpha \beta\left(1+\frac{u_{1}}{u}\right)+\alpha \gamma\left(1+\frac{u_{1}}{u}\right)>0,
$$

$\mathrm{ou}$

$$
\frac{\gamma}{a}+\frac{b u}{\alpha a}>\beta
$$

Logo, para que o ponto de equilíbrio trivial seja localmente e assintoticamente estável, devemos ter:

$$
\beta<\beta^{t h} \equiv \frac{\gamma}{a}+\frac{b u}{\alpha a},
$$

onde $\beta^{\text {th }}$ é a taxa de contato limiar entre vírus e células T-CD4; e é instável para:

$$
\beta>\beta^{\text {th }} \text {. }
$$

A interpretação de $\beta^{\text {th }}$ é dada na próxima secção.

Os coeficientes de $\lambda$ do polinômio entre colchetes de (2.3), designados por $a_{1}$ e $a_{2}$, são tais que $a_{1}^{2}-4 a_{2}>0$. Logo, o equilíbrio trivial é classificado como nódulo. Assim, para $\beta<\beta^{\text {th }}$, todas as trajetórias aproximam de equilíbrio trivial exponencialmente.

O segundo ponto de equilíbrio do sistema (2.1), o ponto de equilíbrio não trivial $P_{*}$, é dado pelos valores:

$$
\left\{\begin{aligned}
v & =\left(\frac{-b u+a \alpha \beta-\alpha \gamma}{b \beta}\right) \\
y & =\frac{b}{(a \beta-\gamma)} \\
z & =\left(\alpha-\frac{b u}{a \beta-\gamma}\right)\left(\frac{1}{u+u_{1}}\right) .
\end{aligned}\right.
$$

Como não tem significado falar em números de vírus e de células negativos, para que o ponto analisado tenha significado biológico, devemos ter:

$$
(v, y, z) \geq 0 .
$$


Assim, o valor de $\beta$ em (2.5), para que condição (2.6) seja satisfeita, deve ser:

$$
\beta \geq \beta^{t h}
$$

onde $\beta^{\text {th }}$ é dada pela equação (2.4).

A estabilidade do equilíbrio não trivial $P_{*}$ é determinada pelos autovalores associados à matriz Jacobiana $J$ calculada com as coordenadas dadas em (2.5), dada por

$$
J=\left(\begin{array}{ccc}
-\left(b+\frac{b \gamma}{a \beta-\gamma}\right) & -\gamma\left(\frac{-b u+a \alpha \beta-\alpha \gamma}{b \beta}\right) & a\left(u+u_{1}\right) \\
-\frac{b \beta}{a \beta-\gamma} & -\left(u+\frac{-b u+a \alpha \beta-\alpha \gamma}{b}\right) & 0 \\
\frac{b \beta}{a \beta-\gamma} & \frac{-b u+a \alpha \beta-\alpha \gamma}{b} & -\left(u+u_{1}\right)
\end{array}\right)
$$

cujos valores são soluções da equação

$$
\begin{aligned}
& \lambda^{3}+\left[b+\left(u+u_{1}\right)+\frac{\alpha}{b}(a \beta-\gamma)+\frac{b \gamma}{a \beta-\gamma}\right] \lambda^{2} \\
& +\left[\left(u+u_{1}\right) \frac{\alpha}{b}(a \beta-\gamma)+\frac{b \gamma u}{a \beta-\gamma}+\alpha(a \beta-\gamma)\right] \lambda \\
& +\left(u+u_{1}\right)[b u-\alpha(a \beta-\gamma)]=0 .
\end{aligned}
$$

Com respeito ao polinômio (2.3), conseguiríamos obter analiticamente os autovalores, porém havíamos aplicado o critério de Routh-Hurwitz de ordem 2. Porém no polinômio acima de terceiro grau, devemos aplicar o critério de Routh-Hurwitz de ordem 3, pois não foi possível obter analiticamente um ou mais autovalores. Segundo o critério de Routh-Hurwitz, para que o polinômio de ordem $3, \lambda^{3}+a_{1} \lambda^{2}+a_{2} \lambda+a_{3}=0$, tenha todas as raízes com parte real negativa, devemos ter: $a_{1}>0, a_{3}>0$ e $a_{1} a_{2}>a_{3}$.

Aplicando para polinômio (2.7) a primeira condição de Routh-Hurwits, $a_{1}>0$, tem-se

$$
b+\left(u+u_{1}\right)+\frac{\alpha}{b}(a \beta-\gamma)+\frac{b \gamma}{a \beta-\gamma}>0
$$

A equação acima é satisfeita para $\beta>\frac{\gamma}{a}$, que é a mesma condição para que $y$ tenha significado biológico.

Para $a_{3}>0$ temos:

$$
\left(u+u_{1}\right)[b u-\alpha(a \beta-\gamma)]>0 .
$$

Como $\left(u+u_{1}\right)>0$, logo:

$$
b u-\alpha(a \beta-\gamma)>0
$$

e obtemos:

$$
\beta>\beta^{\text {th }} \text {. }
$$

O último critério de Routh-Hurwitz é dado por $a_{1} a_{2}>a_{3}$. Antes, porém, vamos simplificar o coeficiente $a_{2}$ para facilitar as contas. A primeira simplicação consiste em definir $a \beta-\gamma=x$, que, substituído em $a_{2}$, resulta:

$$
\left(u+u_{1}\right)\left(\frac{\alpha}{b} x\right)+\alpha x+\frac{b \gamma u}{x} .
$$


Dessa forma a condição $a_{1} a_{2}>a_{3}$ pode ser re-escrito como:

$$
\left[b+\left(u+u_{1}\right)+\frac{b \gamma}{x}+\frac{\alpha x}{b}\right]\left[\left(u+u_{1}\right)\left(\frac{\alpha}{b} x\right)+\alpha x+\frac{b \gamma u}{x}\right]>\left(u+u_{1}\right)(\alpha x-b u) .
$$

Expandindo o primeiro membro, temos:

$$
\begin{aligned}
& \left(u+u_{1}\right)\left[\frac{\gamma b u}{x}+\gamma \alpha+2 \alpha x+\frac{\alpha x}{b}\left(u+u_{1}+\frac{\alpha x}{b}\right)\right] \\
& +\frac{b^{2} \gamma u}{x}+\alpha x b+\frac{b^{2} \gamma^{2} u}{x^{2}}+\alpha b \gamma+\alpha \gamma u+\frac{\alpha^{2} x^{2}}{b}>\left(u+u_{1}\right)(\alpha x-b u),
\end{aligned}
$$

ou, equivalentemente:

$$
\begin{aligned}
& \left(u+u_{1}\right)\left[\frac{\gamma b u}{x}+\gamma \alpha+\alpha x+\frac{\alpha x}{b}\left(u+u_{1}+\frac{\alpha x}{b}\right)+b u\right] \\
& +\frac{b^{2} \gamma u}{x}+\alpha x b+\frac{b^{2} \gamma^{2} u}{x^{2}}+\alpha b \gamma+\alpha \gamma u+\frac{\alpha^{2} x^{2}}{b}>0 .
\end{aligned}
$$

O único termo que possui sinal negativo é o termo em $x, x=a \beta-\gamma$. Logo $a_{1} a_{2}>a_{3}$ é satisfeito com folga se

$$
\beta>\frac{\gamma}{a}
$$

mesmo valor encontrado para $a_{1}>0$.

Concluindo, para que o ponto não trivial seja estável, devemos ter:

$$
\beta>\beta^{t h} \equiv \frac{\gamma}{a}+\frac{b u}{a \alpha} .
$$

Observe que, se $\beta>\beta^{\text {th }}$, então $\beta>\frac{\gamma}{a}$ é automaticamente satisfeita. Assim, se o coeficiente independente de $\lambda, a_{3}$, for positivo, então o ponto de equilíbrio não trivial é localmente e assintoticamente estável [9].

O equilíbrio trivial $P_{0}$, dada pelas coordenadas da equação (2.2), é localmente e assintoticamente estável se $\beta<\beta^{\text {th }}$; e instável se $\beta>\beta^{\text {th }}$. Por outro lado, equilíbrio não trivial $P_{*}$, dada pelas coordenadas da equação (2.5), é localmente e assintoticamente estável se $\beta>\beta^{\text {th }}$. Assim a bifurcação ocorre no valor $\beta=\beta^{\text {th }}$.

\section{Discussão e Conclusão}

A imunologia tem avançado muito na descrição da interação do SI com elementos estranhos que invadem o organismo. A forma de resposta do organismo varia de acordo com o invasor (parasita). Mesmo dentro da classe de micro-parasitas, a resposta do SI difere em se tratando de bactérias, fungos e vírus. Temos como exemplo o vírus HIV que tem como células alvo os linfócitos T-CD4 para se replicar, enquanto o vírus da varicela-zóster aloja-se em células dos gânglios dos nervos espinais ou cranianos, como uma resposta evasiva a uma ação vigorosa do SI.

Neste trabalho apresentamos um modelo simples que descreve a interação do SI com o vírus HIV, em que o vírus é destruído pelos anticorpos produzidos pelas células B ativadas por células T-CD4 ativadas. O resultado do modelo estabelece que, para

$$
\beta<\beta^{\text {th }}
$$


temos um único ponto de equilíbrio, o trivial, que é estável, ou seja, o sistema imunológico obtém sucesso e o vírus é debelado do organismo pelo SI. Mas, para

$$
\beta>\beta^{t h} \equiv \frac{1}{\frac{a}{\gamma}}+\frac{1}{\frac{a}{b} \times \frac{\alpha}{u}},
$$

surge um outro ponto de equilíbrio estável, o não trivial, sendo que o equilíbrio trivial torna-se instável. Nesse caso o vírus não é debelado pelo organismo e a infecção torna-se crônica.

A análise do modelo foi feita do ponto de vista de capacidade infectiva de vírus (parâmetro $\beta$ ), e não da capacidade de resposta imunológica (parâmetro $\gamma$ ). Todo vírus apresenta estratégias bem definidas para sua perpetuação. Nesse sentido cada vírus estabeleceu modos particulares na questão de invasão do hospedeiro, modo de replicação dentro das células e mecanismos de infectar novos hospedeiros. O vírus HIV (restrito apenas à infecção por ato sexual) infecta o ser humano atravessando barreiras físicas (mucosas dos órgãos sexuais). Mesmo que consiga ultrapassar essa barreira, a maioria de vírus é destruída por uma vigorosa resposta imunológica inata. Porém, quando há feridas nas mucosas, ou a inoculação é extremamente elevada, então o vírus vence as primeiras batalhas facilmente e invade a corrente sangüínea. A essa invasão ocorre a resposta imunlógica adaptativa.

A taxa de contato $\beta$ é um valor populacional, da qual podemos escrever $\beta=$ $\beta^{\prime} N_{v}$, onde $\beta^{\prime}$ é a taxa de contato per-capita e $N_{v}$ é a população total de vírus. Assim, o valor limiar $\beta^{\text {th }}$ traz consigo quantidade mínima inoculada para que nova infecção ocorra. Estudemos até quanto uma resposta imunológica pode debelar uma infecção. A taxa de contato limiar é composta de dois termos. Uma contribuição é

$$
\frac{1}{\frac{a}{b} \times \frac{\alpha}{u}} .
$$

Observe que $\frac{\alpha}{u}$ é a concentração de células-alvo em equilíbrio (capacidade de suporte), e $\frac{a}{b}$ é a quantidade de novos vírus liberados por células infectadas durante o período médio de sobrevivência de vírus $\frac{1}{b}$. Uma vez que novas infecções ocorrem conforme a lei da ação das massas, o produto entre concentrações de vírus oriundos da morte de células infectadas e de células alvo, $\frac{a}{b} \times \frac{\alpha}{u}$, descreve a eficácia do encontro das duas populações e consequente infecção. Por isso, a quantidade de vírus que deve ser inoculada para resultar novas infecções depende inversamente desse produto. Outra contribuição é dada por

$$
\frac{1}{\frac{a}{\gamma}} .
$$

Como $a$ é a quantidade de novos vírus que uma célula infectada produz e $\frac{1}{\gamma}$ é o período médio de tempo de ação de anticorpos para destui-los, $\frac{a}{\gamma}$ pode ser entendido como período médio de tempo necessário para neutralizar todos os novos vírus por anticorpos. Por isso, quanto maior for o tempo necessário para destruição de HIV por anticorpos, menor será a inoculação inicial para promover uma nova infecção.

Resposta imunológica baseada apenas em anticorpos tem forte limitação. Novos vírus são liberados aos milhões, que conseguem infectar células suscetíveis a despeito da ação de anticorpos. Para que infecções virais sejam contidas, as células 
infectadas devem ser eliminadas, pois sem esse mecanismo não será possível debelar infecção, exceto para vírus pouco replicadores. O modelo proposto é uma simplificação da interação entre HIV e sistema imunológico, porém mostra que ação isolada de anticorpos é em geral inócua quando as 'fábricas' de vírus não forem destruídas.

\section{Agradecimento}

Os autores agradecem as sugestões dos revisores anônimos que contribuíram substancialmente no aprimoramento desse trabalho.

Abstract. We develop a simple mathematical model in order to study the interaction between HIV and immune system. In the model we take into account only the action of T CD-4 cells. Based on the bifurcation value, we discuss the possibility of the immune system cleansing the circulating virus.

\section{Referências}

[1] G. Adomian, Y. Cherruault, K. Abbaoui, A Nonperturbative Analytical Solution of Immune Response with Time-delays and Possible Generalization, Math. Comput. Modeling, 24 No. 10 (1996), 89-96.

[2] G. Adomian, "Solving Frontier Problems of Physics: The Decomposition Method", Klumer Publication, 1994.

[3] S. Barrozo, H.M. Yang, C.H. Dezotti, Uma abordagem Matemática em Imunologia. In (Editor chefe H.M. Yang): Notas em Matemática Aplicada, vol.7., pp. 93-117, SBMAC \& FAPESP, São Carlos e São Paulo, 2003.

[4] N. Bellomo, L. Preziosi, Modeling and Mathematical Problems Related to Tumor Evolution and Its Interaction with The immune System, Mathematical and Computer Moddeling, 32 (2000), 413-452.

[5] L. Edelstein-Keshet, "Mathematical Models in Biology", McGraw-Hill, Inc., New York, 1988.

[6] W.C. Ferreira Jr., "Dinâmica de Populações: Modelos Matemáticos, Simulações e Aplicações", Notas de Minicurso, XXI CNMAC, Caxambu, MG, 1998.

[7] W.C. Ferreira Jr., "Notas de Biomatemática", IMECC-UNICAMP, Campinas, 2000.

[8] J.D. Murray, "Mathematical Biology I: An Introduction", Ed. Springer, 1993.

[9] H.M. Yang, "Epidemiologia Matemática - Estudos dos Efeitos da Vacinação em Doenças de Transmissão Direta", Ed. UNICAMP \& FAPESP, Campinas, SP, 2001. 
